\title{
Frauendiskriminierung sanktionslos - Europäischer Gerichtshof rügt deutschen Gesetzgeber
}

"Frauendiskriminierung kein Kavaliersdelikt $« .{ }^{1}$ Mit solchen und ähnlich euphorischen Kommentaren wurde die Entscheidung des Gerichtshofes der Europäischen Gemeinschaften (EuGH) in Luxemburg vom 10. April $1984^{2}$ zu der Frage, inwieweit das deutsche "Gesetz über die Gleichbehandlung von Männern und Frauen am Arbeitsplatz und über die Erhaltung von Ansprüchen bei Betriebsübergang « (arbeitsrechtliches EG-Anpassungsgesetz) vom 13.8. 1980 den Anforderungen des europäischen Rechts genügt, in der Öffentlichkeit aufgenommen.

Vielfältige Erfahrungen mit Recht und Justiz haben Frauen gelehrt, gegenüber solchen »Rechtsfortschritten « skeptisch zu sein ${ }^{4}$, zu fragen, inwieweit hier nicht - wie schon so oft in der Vergangenheit - nur symbolisch's den Interessen der Frauen Rechnung getragen wurde. Die Entscheidung des EuGH ist daher unter dem Gesichtspunkt zu untersuchen, inwieweit sie tatsächlich der einzelnen Frau, die von Diskriminierung im Arbeitsleben betroffen ist, ein konkretes Mehr an Sanktionsmöglichkeiten einräumt.

Der Entscheidung des EuGH liegt folgende Vorgeschichte zugrunde: Der Ministerrat der Europäischen Gemeinschaften erließ im Jahre 1976 die "Richtlinie zur Verwirklichung des Grundsatzes der Gleichbehandlung von Männern und Frauen hinsichtlich des Zugangs zur Beschäftigung, zur Berufsausbildung und zum beruflichen Aufstieg sowie in bezug auf die Arbeitsbedingungen. ${ }^{6}{ }^{6}$ Diese Richtlinie enthielt $u$. a. die Bestimmung, daß die Mitgliedsstaaten verpflichtet sind, diejenigen innerstaatlichen Rechtsvorschriften zu erlassen, die notwendig sind, damit jeder, der sich durch eine Diskriminierung für beschwert hält, »seine Rechte gerichtlich geltend machen kann « (Artikel 6).

Gem. Art. I 89 III EWG-Vertrag ist die Richtlinie "für jeden Staat, an den sie gerichtet wird, hinsichtlich des zu erreichenden Ziels verbindlich, überläßt jedoch den innerstaatlichen Stellen die Wahl der Form und der Mittel«. Der deutsche Gesetzgeber erließ nun aufgrund dieser Richtlinie das erwähnte arbeitsrechtliche EGAnpassungsgesetz. Es enthält u. a. folgende Vorschrift: "Ist ein Arbeitsverhältnis wegen eines von dem Arbeitgeber zu vertretenden Verstoßes gegen das Benachteiligungsverbot des Absatzes I nicht begründet worden, so ist er zum Ersatz des Schadens verpflichtet, den der Arbeitnehmer dadurch erleidet, daß er darauf ver-

I So die Schlagzeile der Berliner $» \operatorname{taz} \kappa$ vom I 1. 4. I984.

2 Die Entscheidung ist im Wortlaut abgedruckt in EuGRZ I984, I ז. Jg. Heft 6-8, S. $217 \mathrm{ff}$.

3 BGBl. I S. 1308.

4 Vgl. U. Gerhard, Über gegenwärtige und historische Erfahrungen der Frauen mit Recht. Vorüberlegungen zu einer Rechtstheorie auch für Frauen, in: Gesellschaft. Beiträge zur Marxschen Theorie I 4, Frankfurt/M. 198 i, S. 140 .

s So die Terminologie von M. Edelmann, der damit die bewußte Scheinlösung gesellschaftlicher Konflikte durch staatliche Institutionen beschreibt, vgl. Politik als Ritual, Frankfurt/M. 1977

6 Richtlinie v. 9. 2. 1976 (Abl. L 39, S. 40). 
traut, die Begründung des Arbeitsverhältnisses werde nicht wegen eines solchen Verstoßes unterbleiben « ( $\$ 6$ 1 1a Abs. 2 Satz I BGB).

Unter anderem wegen dieser Schadensersatzregelung, die der diskriminierten Bewerberin nur die Geltendmachung der unnütz aufgewendeten Bewerbungskosten, nicht aber des entgangenen Lohnes, geschweige denn die Geltendmachung der entgangenen Einstellung oder Beförderung ermöglichte, wurde das EG-Anpassungsgesetz von kritischen Stimmen in der rechtswissenschaftlichen Literatur abgelehnt ${ }^{7}$ und in der Öffentlichkeit als »Portoparagraph « apostrophiert. In einer Sachverständigenanhörung des Bundesinnenministeriums und des Bundesfamilienministeriums im Januar 1982 zu der Frage zukünftiger rechtlicher Antidiskriminierungsmaßnahmen wurde vor allem die Forderung erhoben, das EG-Anpassungsgesetz zu reformieren, d. h. insbesondere es mit einer effektiven Schadensersatzregelung auszustatten. ${ }^{8}$

$\mathrm{Daß}$ die kritischen Stimmen Recht hatten, wurde auch dadurch bestätigt, daß die Kommission der Europäischen Gemeinschaften im Januar 1982 gegen die Bundesrepublik ein Klageverfahren anstrengte mit der Begründung, das deutsche arbeitsrechtliche EG-Anpassungsgesetz genüge nicht den Anforderungen der Richtlinie 76/207 zur Gleichbehandlung von Männern und Frauen im Arbeitsleben. Die jetzige Bundesregierung gab aufgrund dieser Vorwürfe zwar Mißstände zu, räumte jedoch nur ein, "mit besonderer Aufmerksamkeit die Entwicklung beobachten ${ }^{9}{ }^{9}$ u wollen. Im übrigen sei das EG-Anpassungsgesetz besser als sein Ruf. ${ }^{\text {to }}$

Trotz des weithin bekannten Faktums der fortdauernden Diskriminierung von Frauen im Arbeitsleben sind denn auch in der Folgezeit anhängige Schadensersatzklagen von Frauen kaum bekannt geworden. Dies wäre wohl auf absehbare Zeit auch so geblieben, wenn es zwei deutsche Arbeitsgerichte (Hamm und Hamburg) nicht abgelehnt hätten, den erwiesenermaßen diskriminierten Frauen ${ }^{11}$ lediglich die unnütz aufgewendeten Bewerbungskosten zuzugestehen, und wenn die beiden Arbeitsgerichte dem Gerichtshof der Europäischen Gemeinschaften nicht die Frage zur Entscheidung vorgelegt hätten, welche konkreten Auswirkungen die auf Gemeinschaftsebene geltende Richtlinie 76/207 für die innerstaatliche Rechtsfindung hat. Im einzelnen fragten die Arbeitsgerichte: Folgt aus der Richtlinie, daß die Bewerberin bei erwiesener Diskriminierung gegen den Arbeitgeber einen Einstellungsanspruch hat oder - falls man dies verneint - welche konkreten Sanktionen schreibt die Richtlinie sonst vor, und ist sie in der Bundesrepublik unmittelbar geltendes Recht? ${ }^{12}$

In der Entscheidung des Gerichtshofes heißt es dazu:

7 Vgl. die Erörterung von V. Slupik, Der Entwurf eines Gesetzes über die Gleichbehandlung von Männern und Frauen am Arbeitsplatz, KJ H. I/1980, S. 8 ff. und H. Pfarr, Kritik des Entwurfs eines Gesetzes über die Gleichbehandlung von Männern und Frauen am Arbeitsplatz, BlStSozArbR 2/1980, S. I 7 ff.; A. Dix, Wie man ein wirkungsloses Gesetz macht, in: Janssen-Jureit (Hrsg.), Frauenprogramm, Hamburg 1979, S. 354 ff.; W. Adamy/A. Kiewel, Gleichberechtigung durch Gesetz, WSI-Mitteilungen 4/1981, S. 257 ff.

8 Vgl. insbesondere den von den Ministerien zu der Anhörung erarbeiteten Fragenkatalog, der diese Schwerpunktsetzung deutlich werden läßt: Bundesminister für Jugend, Familie und Gesundheit, Bundesminister des Innern (Hrsg.), Sachverständigenanhörung am 21./22. Januar 1982 in Bonn zum Thema: Kann die Situation der Frau durch ein Antidiskriminierungsgesetz verbessert werden? Bonn, 1982, S. $98 \mathrm{ff}$.

$9 \mathrm{Vgl}$. den Bericht der Bundesregierung über die Erfahrungen mit dem die Gleichbehandlung von Männern und Frauen am Arbeitsplatz betreffenden Teil des Arbeitsrechtlichen EG-Anpassungsgesetzes und zu der Frage eventueller Benachteiligungen auch außerhalb des Bereiches des Arbeitslebens v. 31. 3. 1983, BT.-Drs. $10 / 14$, S. 17 f.

10 Ebenda, S. 19.

11 EuGRZ 1984 , S. 217

$12 \mathrm{Zu}$ dem genauen Wortlaut der Fragestellung vg!. EuGRZ 1984, S. 220. 
1. Die Richtlinie 76/207 schreibt nicht vor, als Sanktion für eine wegen des Geschlechts erfolgte Diskriminierung beim Zugang zur Beschäftigung den Arbeitgeber, der Urheber der Diskriminierung ist, zum Abschluß eines Arbeitsvertrags mit dem diskriminierten Bewerber zu verpflichten.

2. Die Richtlinie begründet hinsichtlich der Sanktionen für eine etwaige Diskriminierung keine unbedingte und hinreichend bestimmte Verpflichtung, auf die sich ein einzelner mangels rechtzeitig erlassener Durchführungsmaßnahmen berufen könnte, um aufgrund der Richtlinie eine bestimmte Wiedergutmachung zu erlangen, wenn eine solche Rechtsfolge nach den nationalen Rechtsvorschriften nicht vorgesehen oder zugelassen ist.

3. Die Richtlinie 76/207 überläßt es zwar den Mitgliedstaaten, die Sanktion für einen Verstoß gegen das Diskriminierungsverbot unter den verschiedenen Möglichkeiten auszuwählen, die zur Verwirklichung des Ziels der Richtlinie geeignet sind; entscheidet sich ein Mitgliedstaat jedoch dafür, als Sanktion für einen Verstoß gegen dieses Verbot eine Entschädigung zu gewähren, so muß diese jedenfalls, damit ihre Wirksamkeit und ihre abschreckende Wirkung gewährleistet sind, in einem angemessenen Verhältnis zu dem erlittenen Schaden stehen und somit über einen rein symbolischen Schadensersatz wie etwa die bloße Erstattung der Bewerbungskosten hinausgehen. Es ist Sache des nationalen Gerichts, das zur Durchführung der Richtlinie erlassene Gesetz unter voller Ausschöpfung des Beurteilungsspielraums, den ihm das nationale Recht einräumt, in Übereinstimmung mit den Anforderungen des Gemeinschaftsrechts auszulegen und anzuwenden. ${ }^{13}$

Mit dieser Entscheidung hielt sich der EuGH im Rahmen seiner bisherigen Rechtsprechung, nach der sich ein Rechtsanspruch des Einzelnen unmittelbar aus sekundärem Gemeinschaftsrecht in Form der »Richtlinie « nur dann ergibt, wenn sie hinreichend konkretisiert ist, d. h. das Ermessen des Mitgliedsstaates bei der Ausfüllung durch innerstaatliches Recht gleichsam auf null geschrumpft ist. ${ }^{14}$ Eine weitergehende Auslegung des Art. I 89 EWG-Vertrag entspräche auch nicht mehr dem Wortlaut der Vorschrift, wonach nur sekundäres Gemeinschaftsrecht in Form der »Verordnung u unmittelbar in jedem Mitgliedsstaat gilt.

Fraglich erscheint allerdings, ob der EuGH diese Grundsätze in dem hier interessierenden Fall auch konsequent angewandt hat. Art. 6 der genannten Richtlinie zur Verwirklichung der Gleichberechtigung im Arbeitsleben verlangt ausdrücklich, daß der Beschwerte "seine Rechte gerichtlich geltend machen kann", und nicht, daß Diskriminierungen irgendwie wirksam geahndet werden müssen, was dann auch die Möglichkeit beinhalten würde, sie mit staatlichen Strafsanktionen zu belegen. Ihr Recht, nicht diskriminiert zu werden, kann eine Klägerin - wenigstens dann, wenn sie, wie in dem vom EuGH zu entscheidenden Fall, wegen der Diskriminierung nicht eingestellt wurde - denknotwendig wirksam nur im Wege des Einstellungsanspruches oder - als milderes Mittel - durch den Ersatz des entgangenen Lohnes geltend machen.

Dieser im Wortlaut der Richtlinie angelegten Auslegung als konkrete Handlungsanweisung an den innerstaatlichen Gesetzgeber ist der EuGH jedoch nicht gefolgt und konnte daher auch den Schritt, den Klägerinnen unmittelbar Rechtsansprüche einzuräumen, nicht vollziehen. Er hat den Kelch damit an die innerstaatlichen Gerichte zurückgegeben. Die Arbeitsrichter in Hamm und Hamburg werden nun entscheiden müssen, wie sie der Auffassung des EuGH Rechnung tragen wollen, daß der bei einer Diskriminierung zu zahlende Schadensersatz abschreckende Wirkung haben und in einem angemessenen Verhältnis zu dem erlittenen Schaden stehen muß. Diese Entscheidung wird um so schwerer sein, als der Zuspruch solchen Schadensersatzes, der über die Erstattung von Bewerbungskosten hinausgeht, eben nicht »unter voller Ausschöpfung des Beurteilungsspielraumes «, ${ }^{\text {1s }}$ den $\$ 6$ I I a BGB einräumt, erfolgen könnte, sondern eindeutig nur contra legem möglich wäre; denn in der Vorschrift

13 EuGH, ebd., S. 217.

14 Vgl. Rs. 41/74, (Van Duyn) Slg. 1974, S. $1337 \mathrm{ff}$.

15 Vgl. Zif. 3 des Tenors der Entscheidung. 
geht es lediglich um denjenigen Schaden, den der Arbeitnehmer dadurch erleidet, daß er darauf vertraut, die Bewerbung werde nicht von vornherein sinnlos sein. ${ }^{16}$

Diese Tatsache ist dem EuGH aber offenbar bei seiner Urteilsfindung entgangen, obwohl auch die beiden deutschen Arbeitsgerichte diese Auffassung ersichtlich vertraten; im übrigen wäre ihre Vorlage sonst auch wenig sinnvoll gewesen, denn man hätte der eigenen Rechtsauffassung von der Tragweite des europäischen Rechts auch durch eine Anwendung entsprechender deutscher Rechtsgrundsätze im Rahmen der Auslegung des $\$ 6$ I гa BGB Rechnung tragen können. Diese »Täuschung des EuGH mag aber dadurch zu erklären sein, daß die Bundesregierung im Verfahren die Auffassung vertreten hat, $\$ 6$ I ra BGB schließe die Anwendung der allgemeinen Schadensersatzregelungen nicht notwendig aus ${ }^{17}$, eine Meinung, die um so erstaunlicher anmutet, als die Bundesregierung in ihrem Erfahrungsbericht zum EG-Anpassungsgesetz aus dem Jahre 1983 ausdrücklich feststellt, der Ersatz des positiven Interesses (z. B. entgangener Lohn) in Diskriminierungsfällen begegne "ganz erheblichen rechtlichen wie auch praktischen Bedenken $«{ }^{18}$ Der EuGH als Organ der Gemeinschaft darf seinerseits innerstaatliche Rechtsgrundsätze nicht interpretieren, sondern muß sie in der Auslegung hinnehmen, die ihnen die innerstaatlichen Instanzen gegeben haben. ${ }^{19}$ Insofern konnte der EuGH sich auch nicht über die von der Bundesregierung vertretene falsche Auffassung, $\mathbb{\$}$ 6 I a BGB lasse eine Anwendung der allgemeinen Schadensersatzgrundsätze zu, hinwegsetzen.

Die Bedeutung der Entscheidung des Europäischen Gerichtshofes liegt daher weniger im juristischen als im politischen Bereich. Dies hat sicher auch die Prozeßbevollmächtigte einer der Klägerinnen, die Hamburger Arbeitsrechtlerin Heide Pfarr so eingeschätzt, die unmittelbar nach dem Bekanntwerden der Entscheidung den Gesetzgeber aufrief, umgehend im Sinne der Entscheidung tätig zu werden.

Angesichts der bisherigen ignoranten Haltung der Bundesregierung gegenüber einer Reform des arbeitsrechtlichen EG-Anpassungsgesetzes ${ }^{20}$, ihrer unüberhörbaren Ideologisierung der Mütterlichkeit und einer konsequenten Vermeidung jeglicher zusätzlicher finanzieller Belastungen der Unternehmen, muß darauf geachtet werden, daß die juristische Schützenhilfe, die der Gerichtshof der europäischen Gemeinschaften den Frauen gegeben hat, nicht ungenutzt bleibt, und die Forderungen an den Gesetzgeber nicht ungehört verhallen; sonst bleibt Frauendiskriminierung eben doch ein Kavaliersdelikt.

Kristina Garbe-Emden

16 Das AG Hamm hat inzwischen am 6.9.1984 in Konsequenz der Entscheidung des EuGH den beiden Klägerinnen Schadensersatz in Höhe von jeweils DM $21000-$ das entspricht sechs Monatsgehältern der angestrebten Sozialarbeiterinnenstellen - gegen das beklagte Land Nordrhein-Westfalen zugesprochen

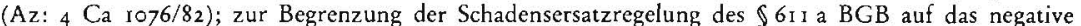
Interesse vgl. V. Slupik, Der Entwurf eines Gesetzes über die Gleichbehandlung von Männern und Frauen am Arbeitsplatz, KJ H. I/ 1980, S. 63.

17 EuGRZ 1984, S. 222.

18 BT.-Drs. $10 / 14$, S. 17

I9 B. Beutler/R. Bieber/J. Pipkorn/J. Streil, Die Europäische Gemeinschaft - Rechtsordnung und Politik, 2. Aufl., Baden-Baden, 1982, S. 195.

20 Ein entsprechender Gesetzesentwurf wurde von der SPD bereits im Jahre 1983 im Bundestag eingebracht; vgl. "Entwurf eines Gesetzes zur Sicherung der Glcichbehandlung von Männern und Frauen am Arbeitsplatz«, BT.-Drs. 10/56 v. I 5. 6. 1983 und mit gleicher Zielsetzung den Gesetzesantrag des Landes Hessen im Bundesrat, BR.-Drs. $180 / 83$ v. 20. 4. 1983 . 
In dem Artikel von Helmut Kramer »Oberlandesgerichtspräsidenten und Generalstaatsanwälte als Gehilfen der NS- Euthanasie« (KJ H. I/1984, S. 25 ff.) ist erfreulicherweise von dem »offiziellen Stop « der T 4 -Aktion vom August 194 I gesprochen worden. Erfreulich deshalb, weil in vielen Berichten über die sogenannte »Euthanasie e behauptet wird, damals sei ein wirklicher Stop der Morde erreicht worden. Tatsächlich sind von diesem Datum ab nur bestimmte Mordmethoden, wie z. B. die bekannt gewordenen Vergasungen, unterblieben. Nach dem August I94 I hat die weitaus überwiegende Anzahl der Ermordungen überhaupt erst stattgefunden, aber nunmehr durch Verweigerung der Ernährung, durch Vergiftungen, durch Unterlassung von medizinischen Behandlungen und andere "unauffällige Mordmethoden. Sogar abgestürzte und gehirngeschädigte deutsche Flieger kamen damals als »nutzlose Esser « um, ebenso zahlreiche leicht Tuberkulöse im deutschen Osten. Über die Ermordung der kranken Flieger wird heute noch von Mitwissern Stillschweigen bewahrt.

Ich selbst bekam die ersten Mitteilungen über die $\mathrm{T}_{4}$-Aktion in USA von Eltern deutscher Refugees, die ihr krankes Kind bei der Auswanderung nach USA in Anstalten hatten zurücklassen müssen. Die Arten der Todesursache und die Wiederholungen fielen mir auf und ich wandte mich an das Reichsinnenministerium mit der Bitte, etwaige neue Bestimmungen über den Gnadentod zu erfahren. Interessanterweise erhielt ich mehrere Aufsätze. Die Postverbindung mit Deutschland funktionierte damals noch in der Zeit bis zum Eintritt der USA in den Krieg im Dezember 1941. Seit damals habe ich mich in Vorbereitung der Nürnberger Prozesse mit diesem Komplex als Erster strafrechtlich befaßt. Als ich den ersten Entwurf einer Anklage gegen den Reichsinnenminister Wilhelm Frick in den USA formulierte, habe ich den Beschuldigten bereits als Mörder der »nutzlosen Esser « bezeichnet, weil er der höchste Verantwortliche für sämtliche öffentlichen Heil- und Pflegeanstalten gewesen war. Als weiteren Verantwortlichen betrachtete ich den »Reichsärzteführer Conti, den ich schon seit meiner Studentenzeit als rechtsradikalen Hetzer kannte.

Nachdem mir im Prozeß vor dem Internationalen Militärtribunal in Nürnberg die Aufgabe zugefallen war, die Anklage gegen Reichsinnenminister Wilhelm Frick zu vertreten, fahndete ich nach dokumentarischen Beweisen für die $\mathrm{T}_{4}$-Aktion. In den Akten der Reichskanzlei entdeckte ich ein Schreiben des Limburger Bischofs Antonius Hilfrich an Hitler. Er wandte sich gegen die längst bekannten EuthanasieErmordungen in der Anstalt Hadamar. Schon die Kinder in der Gegend spielten in den Straßen, daß unbeliebte Kameraden in Hadamar durch den tödlichen Kamin als Rauch herauskämen.

Eine juristische Schwierigkeit bestand darin, daß Ermordungen von Deutschen durch Deutsche als nicht kriegsbedingt zur Zuständigkeit des Internationalen Mili- 